\title{
JSEL: Jamming Skin Enabled Locomotion
}

\author{
E. Steltz and A. Mozeika \\ iRobot Corporation \\ 8 Crosby Dr, Bedford, MA 01730 \\ Email: esteltz, amozeika@irobot.com
}

\author{
N. Rodenberg, E. Brown, and H.M. Jaeger \\ The James Franck Institute \\ The University of Chicago \\ 929 East 57th St, Chicago, IL 60637 \\ Email: rodenberg, embrown, h-jaeger@uchicago.edu
}

\begin{abstract}
A soft, mobile, morphing robot is a desirable platform for traversing rough terrain and navigating into small holes. In this work, a new paradigm in soft robots is presented that utilizes jamming of a granular medium. The concept of activators (as opposed to actuators) is presented to jam and unjam cells that then modulate the direction and amount of work done by a single central actuator. A prototype jamming soft robot utilizing JSEL (Jamming Skin Enabled Locomotion) with external power and control is discussed and both morphing results and mobility (rolling) results are presented. Future directions for the design of a soft, hole traversing robot are discussed, as is the role and promises of jamming as an enabling technology for soft robotics.
\end{abstract}

\section{INTRODUCTION}

The goal of this work funded under the DARPA Chemical Robots program ${ }^{1}$ is to create a flexible, palm-sized robot capable of both autonomous mobility and the more challenging task of hole traversal through an orifice with a diameter 10 times smaller than the characteristic dimension of the robot. The program requirements also imply that the robot might be capable of shape changing or morphing due to the underlying need for flexibility.

The choice of actuator or power source for this robot is a key design element considering the lack of available technology for soft robotics. Conventional robotic actuation technologies (DC motors, pneumatics) are inherently stiff elements or require large stiff pumps in the case of hydraulic or pneumatic solutions. For example, the continuum robot in [1] embodies a soft multiple DOF actuator; however, as can be seen in [2] the pump required for this technology is quite large and would be difficult to make soft. Other pneumatic solutions such as the McKibben actuators in [3] could be appropriate, but for a high DOF system this would require many actuators and therefore many pneumatic lines and valves. Rather, we argue that a fundamental change from standard robotic actuation technology is needed to realize such a soft robot.

Several promising novel actuation schemes have already been used in soft robots. Arguments have been made that dielectric elastomer actuators (or electroactive polymer actuators in general) are promising for a soft robot artificial muscle (see [4] for a good review of the technology in a power delivery context). Dielectric elastomer actuators have already been used to create an earthworm-like robot in [5]. Shape memory alloy actuators are also popular scalable, "soft"

\footnotetext{
${ }^{1}$ This work was funded through a subcontract under the DARPA Chemical Robots program, contract W911NF-08-1-0209
}

actuators and have been used in [6] to make a deformable robot; however, elastic pretensioning is needed for these types of actuators thus requiring stiff frame elements. There are many other proposed actuation technologies that could be appropriate (such as the use of electro-conjugate fluid in [7]). However, all of these actuation technologies are fundamentally mismatched to the problem of a soft, essentially infinite DOF robotic platform because one actuator would be needed for each degree of freedom. To realize true morphing ability and a very high-DOF system, we propose a new paradigm in robotics that distinguishes between actuators and activators. Actuators do work on the environment to enable mobility and morphing, where the activators we define here modulate the actuator output (direction and magnitude). The activators would do no work; rather, they facilitate the actuator to achieve its goals. This allows the use of minimal actuation (hopefully only one actuator) which is more favorable, especially at very small size scales. The specific implementation of this method here uses pneumatic lines with traditional pumps for both the actuator and the activators, but the work is on a trajectory to use alternative mechanisms to trigger the activators.

\section{A. Jamming as a Mobility Mechanism}

Considering the lack of existing technology that can be used to create a soft, hole traversing robot, we are proposing a new application of technology to enable soft robot mobility: jamming. Jamming is the mechanism by which particulate material can transition between a liquid-like and a solid-like state with only a small change in confining volume (see [8] for more information including a jamming phase diagram). To the authors' knowledge, only the work in [9] uses jamming in a robotic system, in that case for haptic feedback. Jamming is commonly experienced in products such as vacuum packed coffee which is shipped in a stiff (solid-like) brick. When this brick is punctured, releasing the confining vacuum, the coffee particles behave liquid-like.

Though jamming itself can do no net external work on the environment to enable mobility, it can modulate the work done by another actuator. For instance, consider the simple case of a ball made up of a jammable material with a balloon in its interior. When the interior balloon is inflated and the jamming medium is in its liquid state, the balloon can do work through the ball to the environment. However, when the jamming medium is in a solid state, the balloon does no work on the environment as long as the jamming medium does not 
yield. This example is in essence the mode in which the robot designed herein operates.

Jamming hence would be the result of the activators. One can imagine having many of these activators throughout a robot that would locally jam the material around them. Though ideally the robot would have many of these local activators resulting in a very high-DOF system, the design proposed in this paper serves to simply demonstrate and motivate the use of jamming as an enabling technology for soft robots and is a first prototype toward mobility and hole traversal.

\section{DESIGN}

To take advantage of jamming as an enabling technology for a soft mobile robot, the topology of Fig. 1 denoted JSEL (Jamming Skin Enabled Locomotion) is introduced. The figure shows the side view of a spherical robot with cellular compartments (cells) around its outer perimeter that are each filled with a jamming (granular) material. The center is filled with a fluid (both compressible and incompressible fluids are acceptable). Morphing capability is easily realized in Fig. 2 by unjamming a subset of the outer cells (in Fig. 2 the unjammed cells are a lighter color) and inflating the central actuator. Volume expansion will occur almost chiefly through deformation of the unjammed cells, thereby changing the shape of the robot. The reader is encouraged to compare this to the case of a soft robot comprised of multiple cells that can be inflated (like balloons). For this simple inflation case, the robot would not be capable of truly deforming its shape; it could only expand about its relaxed shape, and each cell would require an actuator to do work on the environment rather than modulating the work of a central actuator. This is a key difference that jamming introduces.

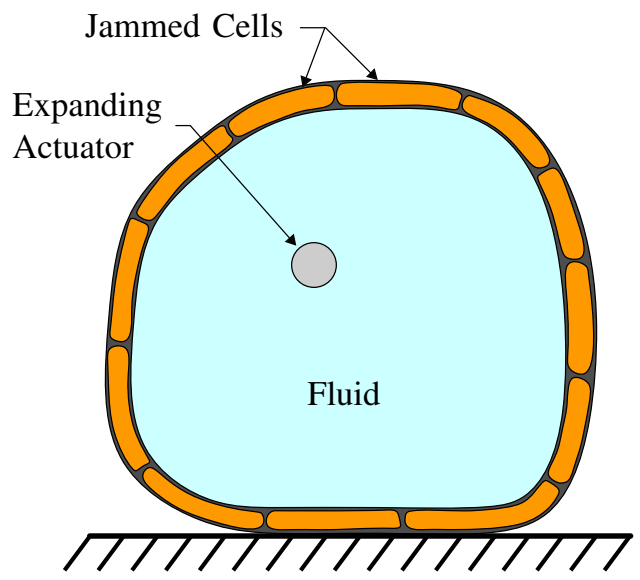

Fig. 1. Side view of proposed jamming based soft robot, all cells jammed, internal actuator deflated.

To approximate a spherical shape, the robot will be constructed as an icosahedron, or a 20 sided solid. An icosahedron was chosen as it is a regular convex polyhedron, or platonic solid, meaning that all its faces, edges, and angles are congruent, making only one cell shape and angle necessary. A CAD model of an icosahedron and the realization of one of its triangular cells are shown in Fig. 3.

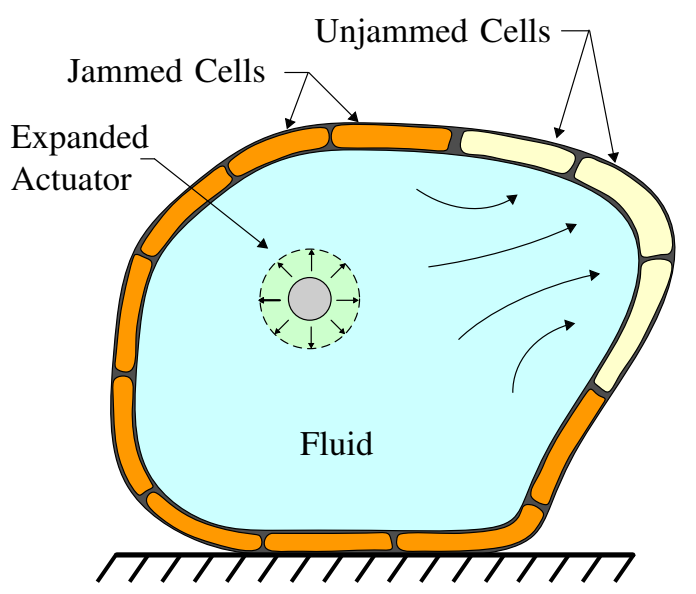

Fig. 2. Side view of proposed jamming based soft robot, 3 cells unjammed, internal actuator inflated partially.

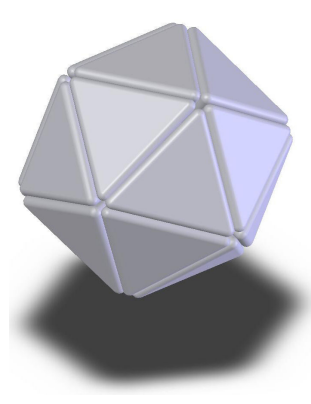

(a)

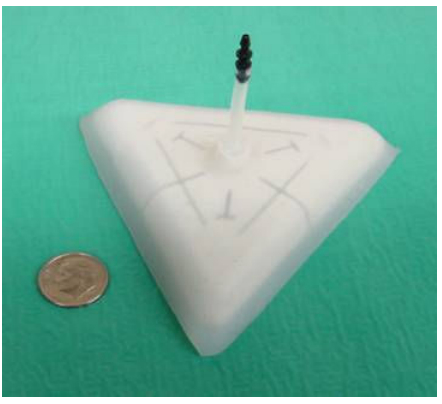

(b)
Fig. 3. A CAD model of the icosahedron (3(a)) and one triangular outer cell (3(b)) shown with a U.S. dime .

\section{FABRICATION}

\section{A. Selection of Jamming Material}

Although many granular materials exhibit jamming behavior, the phenomenon can become more or less pronounced based on the particle size, shape, and compressibility of the particles. To quantify the magnitude of a jammed material's flexural modulus, hollow, thin flexible plastic cylindrical beams were filled with various granular materials and attached to a vacuum pump. The materials of choice are described in Table I and are a variety of sizes and shapes. The beams of each material were supported in a pinned-pinned configuration with their weight as a distributed load and a controllable concentrated force applied downward in the middle of the beam with an Instron. For the zero vacuum case, the distributed load was used as the loading condition; for higher vacuum cases, only the concentrated load was considered since it was much larger than the contribution from the weight of the beam. We then calculate an effective flexural modulus; it is called an "effective" flexural modulus because a jammed material does not behave strictly elastically. Rather, there is a continuous elastic-plastic zone. Therefore, each beam was tested to a set amount of strain (6.9\%) which corresponds to the maximum strain that the weakest beam deflected in its unjammed state $(0$ in. $\mathrm{Hg}$ in the plots that follow). For instance, Fig. 4 shows the 
stress vs strain behavior for the fine $(100 \mu \mathrm{m})$ glass spheres. The dotted lines show the effective modulus if it was a purely elastic material, at $6.9 \%$ strain.

\begin{tabular}{|c|c|c|}
\hline Material & Average Diameter & Shape \\
\hline \hline Large Glass Spheres $^{1}$ & $1.9 \mathrm{~mm}$ & spherical \\
\hline Fine Glass Spheres $^{1}$ & $100 \mu \mathrm{m}$ & spherical \\
\hline Aluminum Oxide $^{2}$ & $100 \mu \mathrm{m}$ & rough and angular \\
\hline Table Salt $^{3}$ & $0.3 \mathrm{~mm}$ & cubic \\
\hline Ground Corn Cob $^{4}$ & $0.8 \mathrm{~mm}$ & rough and angular \\
\hline
\end{tabular}

TABLE I

SIZE AND SHAPE CHARACTERISTICS FOR GRANULAR MATERIALS TESTED. 1:Mo-SCI ClasS IV SODA LIME GLASS SPHERES 2: COTS ALUMINUM OXIDE FOR SAND BLASTING 3:MORTON IODIZED SALT 4:COTS GROUND CORN COB FOR SAND BLASTING

Stress vs Strain for Fine Glass Spheres

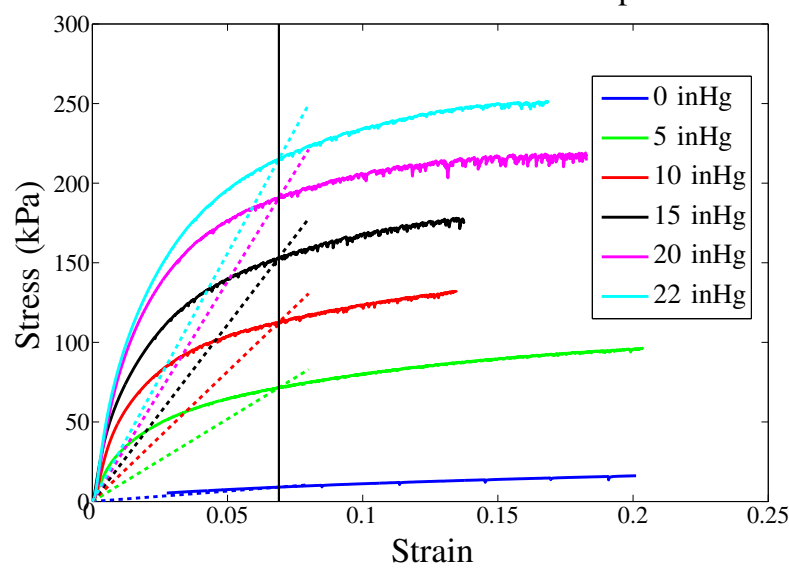

Fig. 4. Stress vs strain for $100 \mu \mathrm{m}$ spherical glass spheres.

This effective flexural modulus vs vacuum level is plotted in Fig. 5 for several materials. Some shape and size characteristics for these granular materials are contained in Table I; the choice of these materials is not meant to be exhaustive in covering all shapes and sizes but to simply give a magnitude quantification for modulus and a quick comparison of the range of magnitudes among several common granular materials. As one can see in the Fig. 5, the effective flexural modulus increases roughly linearly with vacuum level. Here, the table salt has the highest flexural modulus, a desirable characteristic for the material in the jammed state.

However, maximum flexural modulus is not the only design variable of interest in a jamming mobile robot. Also of interest for hole traversal and flexibility is how "liquid-like" the beam is in its non-vacuumed state, a characteristic we found to be expressed in the angle of repose for the granular material. The angle of repose is the angle with which a conical pile of a granular material makes with the horizontal. However, measuring in this way is difficult due to momentum transfer of falling particles to the pile. Rather, we used the method explained in [10] in which particulate matter is drained from a cylinder and the angle of repose is measured from the slope of the material remaining in the cylinder. Table II shows
Effective Flexural Modulus vs Vacuum Level

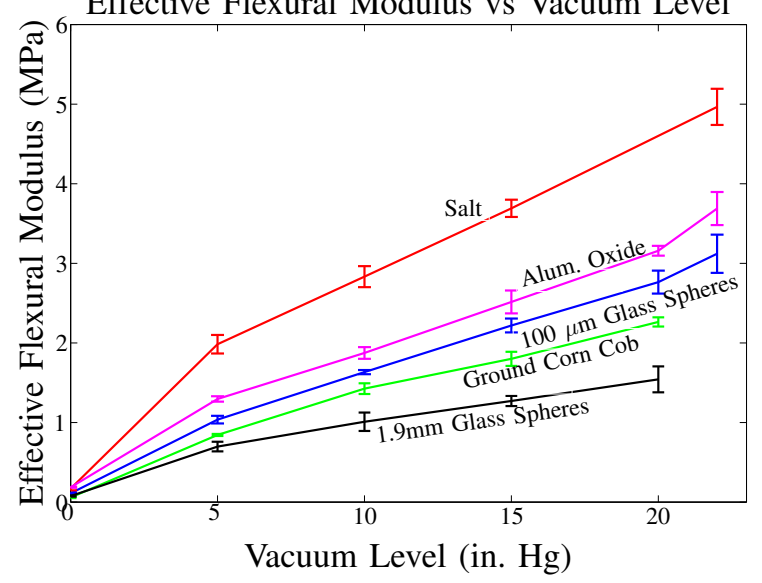

Fig. 5. Effective modulus vs Vacuum Level for various granular material.

\begin{tabular}{|c|c|}
\hline Material & Angle of Repose $\left({ }^{\circ}\right)$ \\
\hline \hline $1.9 \mathrm{~mm}$ Glass Spheres & 21.4 \\
\hline $100 \mu$ m Glass Spheres & 26.6 \\
\hline Aluminum Oxide & 35.9 \\
\hline Table Salt & 37 \\
\hline Ground Corn Cob & 40 \\
\hline
\end{tabular}

TABLE II

AVERAGE ANGLE OF REPOSE FOR VARIOUS GRANULAR SUBSTANCES.

the average angle of repose for the five materials that were measured in the jammed state.

As one can see, if a jamming particulate were chosen simply based on its maximum flexural modulus it can support before yielding, salt would be the optimal particle among those here with $1.9 \mathrm{~mm}$ glass spheres being the least desirable. However, when considering the angle of repose (proportional to the ability of the material to flow), $100 \mu \mathrm{m}$ glass spheres become a desirable choice due to their flexibility in the unjammed state while still competing relatively well with the other particles in the jammed state. Since squeezing through a very small hole is important to the long-term project goals, $100 \mu \mathrm{m}$ glass spheres were utilized as the jamming medium in each icosahedron cell.

\section{B. Cell Fabrication}

An investment casting (or lost-wax) process was used to create the empty icosahedron cells which were later filled with the jamming medium. A plastic positive of the cavity inside each cell was printed using a $3 \mathrm{~d}$ printer (Dimension Elite from Stratasys) as shown in Fig. 6(a). This positive was fit into a plastic box and silicone rubber (DragonSkin Q from SmoothOn) was poured to make a mold of the inner cavity, shown in Fig. 6(b). The rubber section was removed from the plastic box and a bolt of known length was placed into the hole from the cell cavity plastic positive, shown in Fig. 6(c). Melted wax (Amaco FlexWax) was poured into the rubber mold as shown. This wax positive was then removed from the mold and placed into a plastic mold (shown in Fig. 6(d)) that is larger than the wax positive in all directions by a set wall thickness, in this 
case $2 \mathrm{~mm}$. The known length bolt is used to set the depth of the wax positive in the mold so that the top and bottom wall thickness can be set also to $2 \mathrm{~mm}$. Silicone rubber (EcoFlex 00-30 from Smooth-On) was poured into the empty cavity. A magnet was used to hold the bottom of the bolt flush with the plastic mold since the wax floats up through the silicone rubber. The wax can then be melted out of the center of the newly formed cell after the bolt is removed, forming the empty cell illustrated in Fig. 6(e).

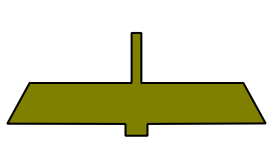

(a)

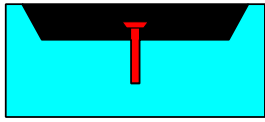

(c)

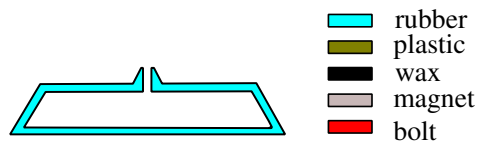

(e)

Fig. 6. The lost wax process used to create empty icosahedron cells out of platinum cure silicon rubber.

The Smooth-On Ecoflex 00-30 is a very soft platinum cure silicone rubber capable of withstanding over $900 \%$ strain before break. This makes the cells very elastic as is demonstrated in Fig. 7. Walls reaching through the triangular cell were also used to keep the granular material from bulging the top and bottom walls out hence leaving a void in the cell (the pattern of the walls are most easily visible in Fig. 3(b). These walls additionally limit the granular medium's ability to flow throughout the cell when under the influence of gravity; this effect is especially problematic when the cell is not completely full of jamming material.

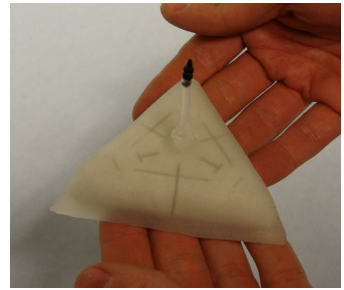

(a)

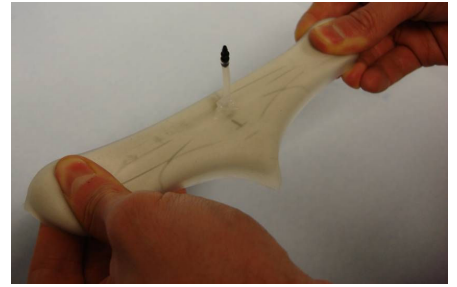

(b)
Fig. 7. A cell relaxed (7(a)) and deformed significantly (7(b)) showing the elasticity of the silicone rubber.

\section{RESULTS}

The JSEL prototype, consisting of 19 jamming cells and one tether pass-through cell (to get the vacuum lines into the robot to its cell inputs) is shown in Fig. 8. The individual cells were adhered together with Smooth-On Ecoflex 5, a fast curing (5 minute) and extremely elastic platinum cure silicone rubber. The tether consists of 19 vacuum lines for the 19 jamming cells and one tube leading into the central core of the actuator. In this prototype, air was used as the fluid diagrammed in Fig. 1, actuated by an external pump. The vacuum lines lead to a valve manifold connected to a vacuum pump, serving here as the activator of jamming. Though this case does not differentiate well between activators and actuators since each has a vacuum line, research is ongoing in making jamming materials that can be activated by effects other than the application of vacuum (such as Electrorheological (ER) or Magnetorheological (MR) fluids). The tether interferes with the robot's mobility to a small extent, though small diameter tubes (1/16") were used to minimize their effect.

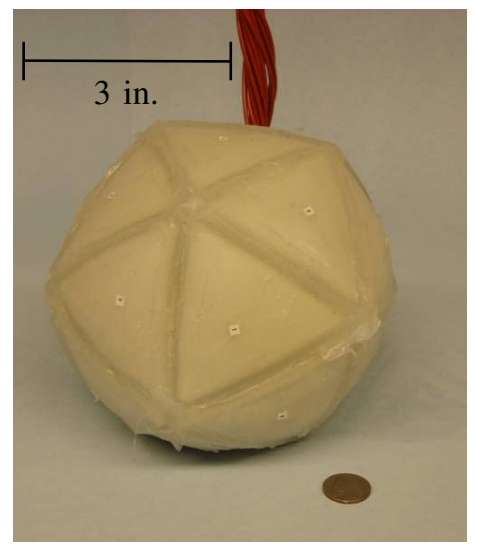

Fig. 8. JSEL prototype alongside a U.S. quarter for scale. The robot is tethered and each of the 20 cells on the robot are numbered as shown.

\section{A. Shape Changing}

Due to the independent addressability of the jamming membrane, the robot can change its shape rather dramatically as is demonstrated in Fig. 9. Fig. 9(a) shows the relaxed shape of the robot. Fig. 9(b) shows the robot inflated with no cells jammed (uniform inflation). The robot can increase its volume significantly before failure. Fig. 9(c) shows the robot shrunk down, accomplished by removing air from the central cavity in the robot.

Fig. 9(d) shows the robot being inflated when all cells are jammed except two (on the right side of the robot). Here one can notice significant shape deformation on one side of the robot. Fig. 9(e) shows the robot with two circular sections pushed out on the right and left side, forming a cylinder shape. This shape is used for the rolling gait discussed in the next section so that the robot rolls straight. Fig. 9(f) shows the robot in the inverse configuration of Fig. 9(e) with the two end caps being drawn back into the main body of the robot with a circular annulus around the robot jammed.

\section{B. Rolling Gait}

The JSEL prototype has the ability to locomote through a rolling gait. Given that the prototype is simply a proof of 


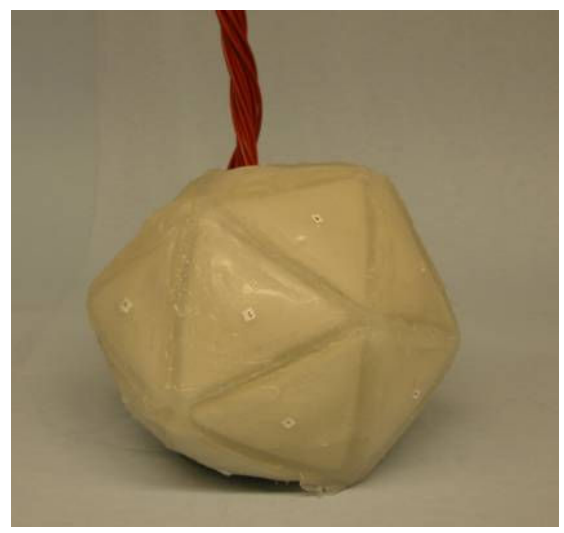

(a)

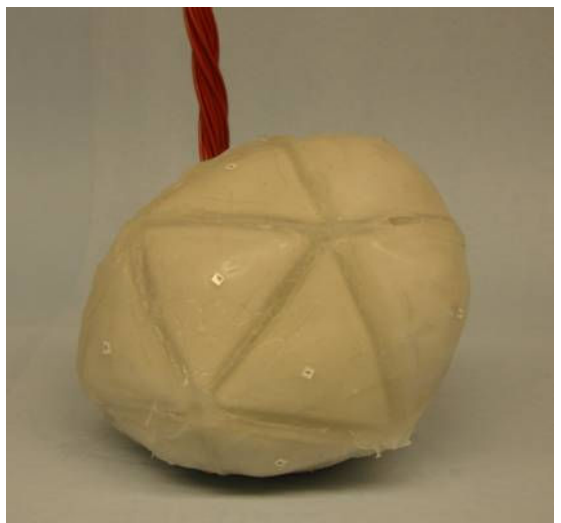

(d)

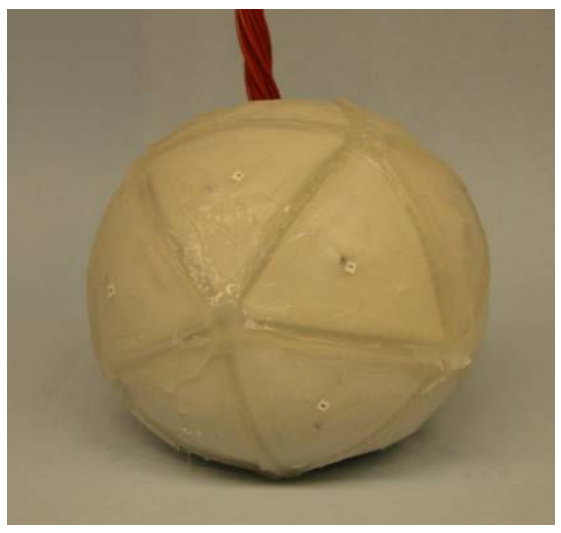

(b)

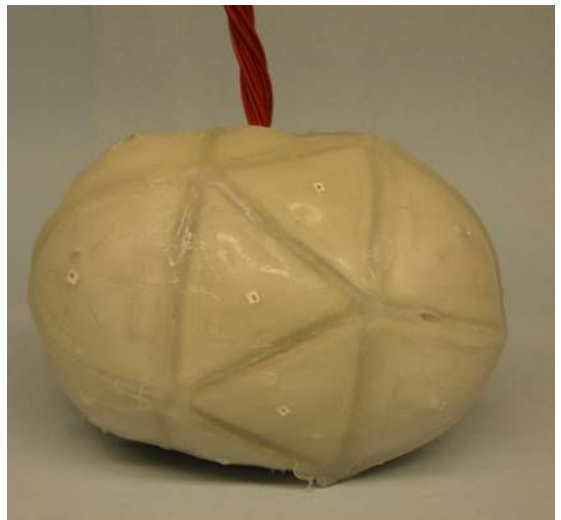

(e)

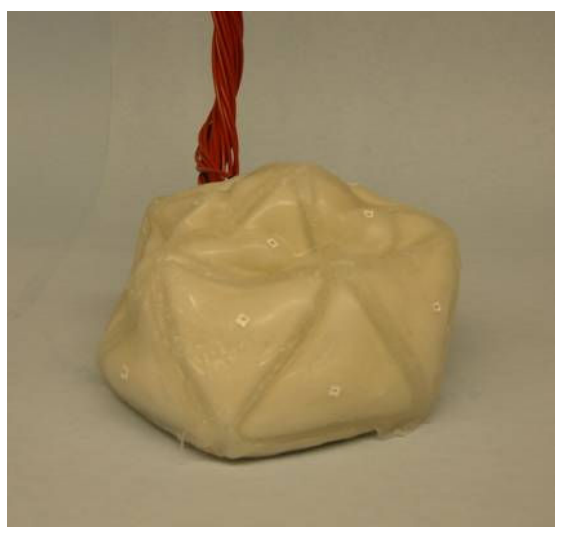

(c)

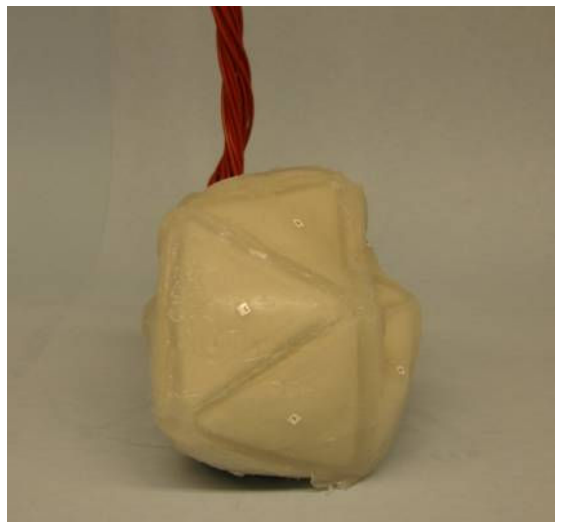

(f)

Fig. 9. Demonstration of the shape changing ability of the JSEL prototype.

concept for using jamming as an enabling mobility mechanism for soft robots, the gait was not optimized in any way. Rather, a very simple and intuitive gait was tried and the robot successfully moved. That gait is as follows: 1) Form a cylinder by jamming an annulus around its center and inflating, thereby pushing out the sides of the robot. 2) Jam the entire robot, then unjam the cell touching the ground at the back of the robot (opposite to the direction of motion) 3) Inflate the central cavity, rolling the robot forward. Repeating this behavior results in continued rolling motion of the robot, as is illustrated in Fig. 10. The tether of the robot was moved along with it by hand so that it would not hold the robot back or move it forward significantly; otherwise, the rolling motion is automated open loop using external pumps.

\section{FUTURE WORK}

The initial JSEL prototype has demonstrated jamming as an effective technology to enable soft, mobile, morphing robots. However, certain limitations have been accepted with the jamming "skin" topology. Given the thickness of the cells on the skin, the amount that these can bend is limited thus limiting the hole traversal ability of the robot. Additionally, the current design does not have an ideal resolution on its outer skin (in other words, more cells are needed) to enable full morphing ability. Also, since the icosahedron is the platonic solid with the largest number of sides, increasing the resolution would require several different shaped skin cells.

\section{A. Future Robot Topology}

Rather than increasing the resolution of the skin design and still having the problem of bending these thick cells through a hole, the design of Fig. 11 is proposed. In this design, many jammable cells are continuously distributed in a lubricating medium, constrained by a thin hyperelastic membrane. Each cell contains an activator that can jam the cell, and the cells have many tendrils to interlock with each other. When each cell is jammed, its tendrils are stiff and interlock with jammed cells around it; when the cells are unjammed, the lubricating medium facilitates easy movement between the cells. Essentially, this is jamming (or interlocking) of selectively jammed cells. Each cell could be connected via elastic wires to control electronics or each could have its own power and communication electronics, communicating via $\mathrm{RF}$ or even via sound through the lubricating fluid.

If an entire section of cells are unjammed and the interior actuator expands, the robot will change its shape and expand out the only unjammed section as shown in Fig. 11. This is similar to the skin cell morphing ability of the current prototype; however, given the small size of the jamming cells and the fact that they are not only on the outside perimeter 


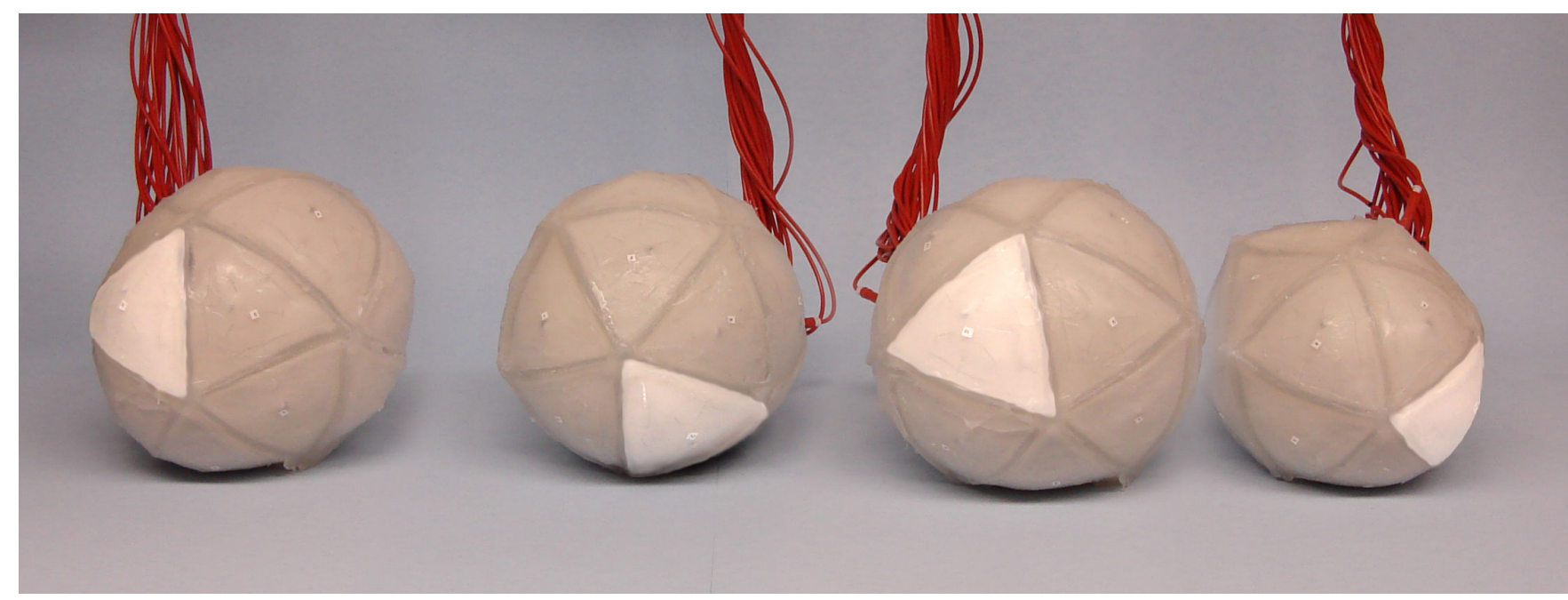

Fig. 10. Composited figures of the JSEL prototype rolling over one revolution. One cell is highlighted to better demonstrate its motion.

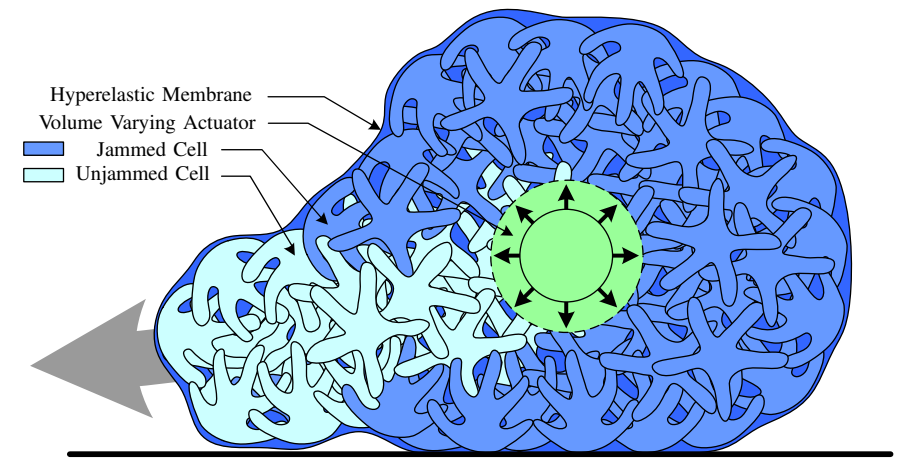

Fig. 11. Diagram of future jamming robot concept.

of the robot gives much greater flexibility and hole traversing ability to the design.

\section{B. Custom Particle Development}

Rather than using off the shelf particles to facilitate jamming, it is not difficult to imagine that custom particle shapes can be engineered to either increase the jamming strength of a material, improve its unjammed flow, make it easier to jam (for example, using less vacuum level), or even to engineer different jamming mechanisms and hence activators. These different jamming mechanisms and activators could include but are not limited to shear thickening or thinning, electrostatically (electrorheological) driven jamming, magnetically (magnetorheological) driven jamming, or even photorheological (light-induced) jamming.

\section{CONCLUSION}

This work is the first demonstration of a completely soft, mobile robot using jamming as an enabling technology. The robot created herein uses the concept of JSEL (Jamming Skin Enabled Locomotion) and is capable of both shape changing and mobility through rolling. It uses only a single actuator to inflate a central bladder in a spherical robot with 19 individually addressable jamming cells. The argument has been made that these jamming cells could be jammed not by an actuator (as is done here with vacuum) but by a low power, small scale activator that could generate jamming with custom particles through electric field, magnetic field, or by various other means. These jamming cells would then modulate the power output of the main actuator as is done in the present robot to create an autonomous, high degree of freedom soft robot.

\section{REFERENCES}

[1] S. Neppalli and B. A. Jones, "Design, construction, and analysis of a contiuum robot," in IEEE/RSJ Int. Conf. on Intelligent Robots and Systems, 2007.

[2] W. McMahan, W. Chitrakan, M. Csencsits, D. Dawson, I. Walker, B. Jones, M. Pritts, D. Dienno, M. Grissom, and C. Rahn, "Field trials and testing of the octarm contiuum manipulator," in IEEE Int. Conf on Robotics and Automation, 2006.

[3] F. Zhao, S. Dohta, T. Akagi, and H. Matsushita, "Development of a bending actuator using a rubber artificial muscle and its application to a robot hand," in SICE-ICASE Int. Joint Conf., 2006.

[4] K. Meijer, M. Rosenthal, and R. Full, "Muscle-like actuators? a comparison between three electroactive polymers," in Smart Structure and Materials 2001: Electroactive Polymer Actuators and Devices. Proc. SPIE, Y. Bar-Cohen, Ed., vol. 4329, 2001, pp. 7-15.

[5] H. Choi, S. Ryew, K. Jung, H. Kim, J. Jeon, J. Nam, R. Maeda, and K. Tanie, "Micro robot actuated by soft actuators based on dielectric elastomer," in IEEE/RSJ Intl. Conf. on Intelligent Robots and Systems, 2002.

[6] Y. Sugiyama and S. Hirai, "Crawling and jumping of deformable soft robot," in IEEE/RSJ Int. Conf on Intelligent Robots and Systems, 2004.

[7] K. Takemura, S. Yokota, and K. Edamura, "A micro artificial muscle actuator using electro-conjugate fluid," in IEEE Int. Conf. on Robotics and Automation, 2005.

[8] A. Liu and S. Nagel, "Jamming is not just cool any more," Nature, vol. 396, p. 21, 1998.

[9] T. Mitsuda, M. Wakabayashi, S. Kuge, and S. Kawamura, "Wearable haptic display by the use of a particle mechanical constraint," in 10th Symp. on Haptic Interfaces for Virtual Envir. and Teleoperator Systs., 2002.

[10] Z. Zatloukal and Z. Sklubalova, "Drained angle of free-flowable powders," Particulate Science and Technology, vol. 26, pp. 595-607, 2008. 